\title{
LOCALLY CONVEX SPACES WITH TOEPLITZ DECOMPOSITIONS
}

\author{
PEDRO J. PAÚL, CARMEN SÁEZ and JUAN M. VIRUÉS
}

Dedicated to Pedro Pérez Carreras on the occasion of his fiftieth birthday

(Received 20 November 1998; revised 3 May 1999)

Communicated by P. G. Dodds

\begin{abstract}
A Toeplitz decomposition of a locally convex space $E$ into subspaces $\left(E_{k}\right)$ with continuous projections $\left(P_{k}\right)$ is a decomposition of every $x \in E$ as $x=\sum_{k} P_{k} x$ where ordinary summability has been replaced by summability with respect to an infinite and row-finite matrix. We extend to the setting of Toeplitz decompositions a number of results about the locally convex structure of a space with a Schauder decomposition. Namely, we give some necessary or sufficient conditions for being reflexive, a Montel space or a Schwartz space. Roughly speaking, each of these locally convex properties is linked to a property of the convergence of the decomposition. We apply these results to study some structural questions in projective tensor products and spaces with Cesàro bases.

1991 Mathematics subject classification (Amer. Math. Soc.): primary 46A11, 46A25, 46A35, 46A45. Keywords and phrases: Decompositions of locally convex spaces, reflexive, Montel and Schwartz spaces, summability and bases, tensor products, sequence spaces.
\end{abstract}

\section{Introduction}

Up to what point can one substitute ordinary summability by a matrix summability method in the definition of a Schauder decomposition and, still, obtain nice results about the locally convex structure of the space in terms of the locally convex structure of its pieces? Our purpose here is to extend to the setting of decompositions defined in terms of matrix summability methods-Toeplitz decompositions-a number of results about the locally convex structure of a space with a Schauder decomposition.

This research has been partially supported by la Consejería de Educación y Ciencia de la Junta de Andalucía and by la Dirección General de Investigación Científica y Técnica, project PB97-0706 (C) 2000 Australian Mathematical Society 0263-6115/2000 \$A2.00+0.00 
In the second section we extend the well-known characterization of semi-reflexive locally convex spaces having a Schauder decomposition [Ka] to the setting of decompositions defined in terms of more general matrix summability methods. The connection between shrinking and $\gamma$-complete decompositions with semi-reflexivity has a long history in basis theory and sequence spaces [Ja, Ka, Sil] and we give appropriate notions within the framework of Toeplitz decompositions. In the third section we extend the well-known Mazur's compactness criterion and apply it to obtain conditions for a space with a Toeplitz decomposition to be a Montel space. In the proof we use a double limit technique that enables us to obtain somewhat simplified (even for the ordinary summability case) proofs. A similar treatment of the quasi-normability and Schwartz property is carried on in the fourth section. These sections can be considered as a continuation of the study started by Díaz and Miñarro [DM1, DM2, Mi] for Fréchet spaces. These results are then applied in the last two sections to study stability questions in projective tensor products and spaces with Cesàro basis. This paper continues our study of locally convex properties of spaces with a Toeplitz basis; previously we have studied similar problems related to completeness [PSV1] and barrelledness [PSV2].

\subsection{Terminology and notation Although our notation and terminology will be} mostly standard, for instance, $\varphi$ is the space of finitely nonzero sequences, $c$ is the space of convergent sequences, $e^{[k]}$ stands for the $k$-th unit sequence (we refer the reader to $[\mathrm{Jr}, \mathrm{PeB}, \mathrm{Si} 2, \mathrm{Ru}, \mathrm{V}]$ or [Wi2]), let us recall a few facts from summability theory. Let $T=\left[t_{n k}\right]$ be an infinite matrix of scalars from the field $\mathbb{K}$ of real or complex numbers. The matrix $T$ is said to be: row-finite if each row of $T$ is in $\varphi$, an $S p_{1}$-matrix if each column of $T$ is convergent to 1 , and reversible if for every sequence $y \in c$ the infinite system of linear equations $T x=y$ has a unique solution. It is well-known [Wi2, 5.4.5-5.4.9] that each row-finite and reversible $T$ has a unique two-sided inverse matrix $T^{-1}$ such that each row of $T^{-1}$ is in $\ell^{1}$ and for each $y \in c$ the unique solution of $T x=y$ is $T^{-1} y$. An important particular case is that of a triangle. Following Wilansky [Wi2], a lower triangular infinite matrix with non-zero diagonal entries is called a triangle. A triangle is always row-finite and reversible, and its inverse is also a triangle. We shall denote by $\Sigma$ the ordinary summability matrix, that is, the triangle with all of its lower triangular entries equal to 1 . More generally, if $A=\left[a_{n k}\right]$ is a row-finite, reversible and regular (that is, a sequence-to-sequence limit preserving) matrix, then $T:=A \Sigma$ is a row-finite, reversible and $S p_{1}$ matrix such that $\sup \left\{\left|t_{n k}\right|: n, k \in \mathbb{N}\right\} \leq\|A\|$, where $\|A\|:=\sup \left\{\sum_{k}\left|a_{n k}\right|: n \in \mathbb{N}\right\}<\infty$ is called the row-norm of $A$. If $A$ is a triangle then $T=A \Sigma$ is also a triangle.

Let $E$ be a locally convex space. We shall denote by $E^{\prime}$ its topological dual and by $\mathscr{U}(E)$ a basis of absolutely convex and closed zero-neighborhoods. Given $U \in \mathscr{U}(E)$ its Minkowski's functional will be denoted by $q_{U}$. (For the standard locally convex 
space terminology we refer the reader to [Jr, Kö, PeB] or [Wil].) The convergence field of a row-finite matrix $T$ in $E$ is the space $c_{T}(E)$ of all sequences $\left(x_{k}\right)$ from $E$ such that the product $T\left(x_{k}\right)$ is a convergent sequence in $E$. The sequences in $c_{T}(E)$ are said to be $T$-convergent. For $\left(x_{k}\right) \in c_{T}(E)$ the limit of the sequence $T\left(x_{k}\right)$ is called the $T$-limit of $\left(x_{k}\right)$ and will be denoted by $T-\lim x_{k}$, in other words

$$
T-\lim x_{k}:=\lim _{n} \sum_{k} t_{n k} x_{k} .
$$

We simply denote by $c_{T}$ the convergence field of $T$ in $\mathbb{K}$. If $T$ is a row-finite and reversible matrix then the norm $\|x\|_{T}:=\|T x\|_{\infty}$ makes $c_{T}$ a Banach space (isomorphic to $c$ ).

DEFINITION. Let $T=\left[t_{n k}\right]$ be a row-finite matrix of scalars. A sequence $\left(P_{k}\right)$ of non-trivial, mutually orthogonal and continuous linear projections defined on a locally convex space $E$ is said to be a Toeplitz decomposition of $E$ with respect to the matrix $T$ or, shortly, a $T$-decomposition of $E$; if

$$
x=T-\lim P_{k} x, \quad \text { for every } x \in E .
$$

Alternatively, if we define the sequence of operators

$$
T_{n}: x \in E \rightarrow T_{n}(x):=\sum_{k} t_{n k} P_{k} x \in E,
$$

then $\left(P_{k}\right)$ is a $T$-decomposition of $E$ whenever $\lim _{n} T_{n} x=x$ for every $x \in E$. Note that $\Sigma$-decompositions are the familiar Schauder decompositions.

It is important to note that the $T_{n}$ 's are not projections in general (they are increasing projections in the case of a Schauder decomposition). However, we do have $T_{n} P_{k}=$ $P_{k} T_{n}=t_{n k} P_{k}$ for all $n, k \in \mathbb{N}$. Note also that the sequence of operators $\left(T_{n}\right)$ is precisely the product $T\left(P_{k}\right)$; hence saying that $\lim _{n} T_{n} x=x$ is the same as saying that the sequence $T\left(P_{k} x\right)$ converges to $x$. Define $E_{k}:=P_{k}(E)$. Since $E_{k}$ does not reduce to the zero subspace and for every $x_{k} \in E_{k}$ we have $x_{k}=\lim _{n} T_{n} x_{k}=\lim _{n} t_{n k} x_{k}$, it follows that $\lim _{n} t_{n k}=1$. Hence, $T$ is an $S p_{1}$-matrix.

Still another way of looking at a Toeplitz decomposition is as follows. Every $E_{k}$ is a complemented subspace of $E$ and we can identify every $x \in E$ with the vector-valued sequence $\left(P_{k} x\right) \in \prod E_{k}$, so that $E$ becomes a linear subspace of $\prod E_{k}$ that, with the topology translated from $E$, has the set of all finite sequences as a dense subspace because $\lim _{n} T_{n} x=x$ for every $x \in E$ and $T$ is row-finite.

We now extend some of the terminology commonly used for Schauder decompositions (see [Ka, Sil, We]). A $T$-decomposition $\left(P_{k}\right)$ of a locally convex space $E$ is said to be: triangular if $T$ is a triangle, regular if $T$ can be factorized as $T=A \Sigma$ for some 
regular matrix $A$, finite-dimensional if every $E_{k}$ is finite-dimensional, equicontinuous if the sequence of operators $\left(T_{n}\right)$ is equicontinuous, and complete (respectively $\beta$ complete, respectively $\gamma$-complete) if for each sequence $\left(x_{k}\right) \in \prod E_{k}$ such that the product $T\left(x_{k}\right)$ is a Cauchy sequence in $E$ (respectively a weakly Cauchy sequence, respectively a bounded sequence) there exists $x \in E$ such that $x_{k}=P_{k} x$ for every $k \in \mathbb{N}$ and, a fortiori, $T\left(x_{k}\right)$ converges to $x$. Note that $\beta$-complete means exactly complete for the weak topology.

Using primes to denote adjoint operators, for every $x \in E$ and every $x^{\prime} \in E^{\prime}$ we can write

$$
\left\langle x, x^{\prime}\right\rangle=\lim _{n}\left\langle T_{n} x, x^{\prime}\right\rangle=\lim _{n} \sum_{k} t_{n k}\left\langle P_{k} x, x^{\prime}\right\rangle=\lim _{n} \sum_{k} t_{n k}\left\langle x, P_{k}^{\prime} x^{\prime}\right\rangle=\lim _{n}\left\langle x, T_{n}^{\prime} x^{\prime}\right\rangle .
$$

This shows that $\left(P_{k}^{\prime}\right)$ is also a $T$-decomposition of $E^{\prime}$ endowed with the weak topology $\sigma\left(E^{\prime}, E\right)$. If we define $E_{k}^{\prime}:=P_{k}^{\prime}\left(E^{\prime}\right)$, then the dual of $E_{k}$ can be identified with $E_{k}^{\prime}$. The computation above also shows that the sequence $\left(T_{n}^{\prime} x^{\prime}\right)$ is $\sigma\left(E^{\prime}, E\right)$-bounded for every $x^{\prime} \in E^{\prime}$. A $T$-decomposition $\left(P_{k}\right)$ of a locally convex space $E$ is said to be shrinking if $\left(P_{k}^{\prime}\right)$ is also a $T$-decomposition of $E^{\prime}$ endowed with the strong topology $\beta\left(E^{\prime}, E\right)$ and is said to be simple if $\left(T_{n}^{\prime} x^{\prime}\right)$ is a $\beta\left(E^{\prime}, E\right)$-bounded sequence for every $x^{\prime} \in E^{\prime}$.

EXAMPLE 1.1. A $K$-space is a locally convex sequence space $\lambda$ containing $\varphi$ such that the $k$-th projection, defined by $\pi_{k}\left(\left(x_{n}\right)_{n}\right):=x_{k} e^{[k]}$, is continuous for every $k \in \mathbb{N}$. A $K$-space $\lambda$ is said to have property $T$ - $A K$ if $x=T-\lim x_{k} e^{[k]}$ for every sequence $x=\left(x_{k}\right) \in \lambda$. Thus, a sequence space $\lambda$ has property $T-A K$ if and only if the sequence $\left(\pi_{k}\right)$ is a (one-dimensional) $T$-decomposition of $\lambda$ or, in other words, if the sequence of operators defined by $\tau_{n}:=\sum_{k} t_{n k} \pi_{k}$, that is $\left(\tau_{n}\right):=T\left(\pi_{k}\right)$, satisfies $x=\lim _{n} \tau_{n}\left(x_{k}\right)$ for every sequence $x=\left(x_{k}\right) \in \lambda$ (see [Bu1, Bu2] or [Me]). (When dealing with scalar sequence spaces, we shall keep the notations $\left(\pi_{k}\right)$ and $\left(\tau_{k}\right)$ throughout the paper). In particular, $\lambda$ has property $\Sigma-A K$ means precisely that $\left(e^{[k]}\right)$ is a Schauder basis of $\lambda$.

We shall be interested in matrices $T$ such that $c_{r}$ has property $T-A K$. These matrices were characterized by Buntinas [Bu2, Theorems 8-10].

THEOREM 1.2 (Buntinas's Theorem). Let $T$ be row-finite and reversible $S p_{1}$-matrix. Then the following conditions are equivalent:

(1) The sequence of coordinate projections $\left(\pi_{k}\right)$ is a T-decomposition of $c_{T}$.

(2) The sequence of operators $\left(\tau_{n}\right)$ is equicontinuous on $c_{T}$.

(3) If we denote $T^{-1}$ by $\left[s_{n k}\right]$ then $\sup \left\{\sum_{j}\left|\sum_{k} t_{m k} t_{n k} s_{k j}\right|: m, n \in \mathbb{N}\right\}<\infty$.

(4) The dual $\left(c_{T}\right)^{\prime}$ can be identified with the multiplier space of $c_{T}$ into $c_{T}$ formed by the sequences $y$ such that the coordinatewise product $x y$ is in $c_{T}$ for every $x \in c_{T}$ and, in this case, the bilinear form of the dual pair is given by $\langle x, y\rangle=T-\lim x y$. 
The first known non-trivial examples of matrices $T$ such that $c_{T}$ has property $T$ - $A K$ are the series-to-sequence Cesàro matrices of order $\alpha \geq 0$; this was proved by Zeller [Ze]. Therefore, to avoid clumsy repetitions, a row-finite and reversible $S p_{1}$-matrix $T$ such that $c_{T}$ has property $T-A K$ will be called a Zeller-Buntinas matrix. For such a matrix $T$ we will consider its equicontinuity constant defined by $b_{T}:=\sup _{n}\left\|\tau_{n}\right\|$, where $\left\|\tau_{n}\right\|$ is the norm of $\tau_{n}$ as a bounded operator from the Banach space $c_{T}$ into itself. Note also that if $T$ is a Zeller-Buntinas matrix then $c_{T}$ is a sum space in the sense of Ruckle [Ru].

\section{Reflexive spaces}

As promised, we start by extending a well-known characterization of semi-reflexive locally convex spaces having a Schauder decomposition [Ka] to the setting of Toeplitz decompositions.

THEOREM 2.1. Let $\left(P_{k}\right)$ be a T-decomposition of a locally convex space $E$. Then the following conditions are equivalent:

(1) $E$ is semi-reflexive.

(2) $\left(P_{k}\right)$ is $\gamma$-complete and shrinking, and each $E_{k}$ is semi-reflexive.

(3) $\left(P_{k}\right)$ is $\beta$-complete and shrinking, and each $E_{k}$ is semi-reflexive.

ProOF. (1) implies (2): Each $E_{k}$ is semi-reflexive because it is a complemented subspace of $E$. To prove that $\left(P_{k}^{\prime}\right)$ is a $T$-decomposition of $E^{\prime}\left[\beta\left(E^{\prime}, E\right)\right]$, start by noting that each $P_{k}^{\prime}$ is weak-to-weak continuous, hence it is also strong-to-strong continuous [Jr, 8.6], [Wil, p. 168]. Since $E$ is semi-reflexive it follows [Kö, Section 23.3.(4)] that $E^{\prime}\left[\beta\left(E^{\prime}, E\right)\right]$ is a barrelled space with dual $E$. This fact implies that $\bigcup_{m \in \mathbb{N}}\left(E_{1}^{\prime}+E_{2}^{\prime}+\cdots+E_{m}^{\prime}\right)$ is dense in $E^{\prime}\left[\beta\left(E^{\prime}, E\right)\right]$ because it is a convex set which is dense in $E^{\prime}\left[\sigma\left(E^{\prime}, E\right)\right]$ and it also implies, on the other hand, that the sequence $\left(T_{n}^{\prime}\right)$ is $\beta\left(E^{\prime}, E\right)$-equicontinuous because, as pointed out above, $\left(T_{n}^{\prime} x^{\prime}\right)$ is $\sigma\left(E^{\prime}, E\right)$-bounded for every $x^{\prime} \in E^{\prime}$. Now the fact that $T$ is a $S p_{1}$-matrix implies that the $\beta\left(E^{\prime}, E\right)$-equicontinuous sequence $\left(T_{n}^{\prime}\right)$ has the property that $\left(T_{n}^{\prime} x^{\prime}\right)$ converges strongly to $x^{\prime}$ for every $x^{\prime}$ in the $\beta\left(E^{\prime}, E\right)$-dense set $\bigcup_{m \in \mathbb{N}}\left(E_{1}^{\prime}+E_{2}^{\prime}+\cdots+E_{m}^{\prime}\right)$. It follows that $\left(T_{n}^{\prime} x^{\prime}\right)$ converges strongly to $x^{\prime}$ for every $x^{\prime} \in E^{\prime}$ [Kö, Section 39.4.(1)], and this is the same as saying that $\left(P_{k}^{\prime}\right)$ is a $T$-decomposition of $E^{\prime}\left[\beta\left(E^{\prime}, E\right)\right]$. It remains to show that $\left(P_{k}\right)$ is $\gamma$-complete or, equivalently, that whenever $\left(x_{k}\right) \in \prod E_{k}$ is such that $T\left(x_{k}\right)$ is bounded then there exists $x \in E$ such that $P_{k} x=x_{k}$ for each $k \in \mathbb{N}$. Every such sequence $\left(x_{k}\right)$ defines a linear functional $f$ on $\bigcup_{m \in \mathbb{N}}\left(E_{1}^{\prime}+E_{2}^{\prime}+\cdots+E_{m}^{\prime}\right)$ as follows. Take $f\left(x^{\prime}\right):=\left\langle x_{k}, x^{\prime}\right\rangle$ whenever $x^{\prime} \in E_{k}^{\prime}$ and $k \in \mathbb{N}$. Since the sum $E_{1}^{\prime}+E_{2}^{\prime}+\cdots+E_{m}^{\prime}$ is actually a direct sum, because the spaces $E_{k}$ 's are the ranges of 
mutually orthogonal and continuous linear projections, it follows that we may extend $f$ to the whole of $\bigcup_{m \in \mathbb{N}}\left(E_{1}^{\prime}+E_{2}^{\prime}+\cdots+E_{m}^{\prime}\right)$ by linearity. Let us see now how we may extend $f$ to the whole of $E^{\prime}$. Let $B$ be the polar set of $\left\{T\left(x_{k}\right)\right\}$ in $E^{\prime}$ and fix $x^{\prime}=\sum_{k=1}^{m} x_{k}^{\prime} \in B \cap \sum_{k=1}^{m} E_{k}^{\prime}\left(\right.$ with $x_{k}^{\prime} \in E_{k}^{\prime}$ ). Then

$$
1 \geq \sup _{n}\left|\left\langle\sum_{k} t_{n k} x_{k}, x^{\prime}\right\rangle\right|=\sup _{n}\left|\sum_{k=1}^{m} t_{n k}\left\langle x_{k}, x_{k}^{\prime}\right\rangle\right| .
$$

Take the limit as $n \rightarrow \infty$ to deduce, using that $T$ is an $S p_{1}$-matrix, that $\left|f\left(x^{\prime}\right)\right| \leq 1$. By the Hahn-Banach theorem, $f$ can be extended to all of $E^{\prime}$ verifying that $\left|f\left(x^{\prime}\right)\right| \leq 1$ for all $x^{\prime} \in B$. Since $E$ is semi-reflexive $f$ must coincide with the linear form defined on $E^{\prime}$ by some $x \in E$ and this element $x$ obviously verifies $P_{k} x=x_{k}$ for all $k \in \mathbb{N}$.

(2) implies (3) is trivial, so let us prove that (3) implies (1). Take $x^{\prime \prime} \in E^{\prime \prime}$. Since every $E_{k}$ is semi-reflexive we have that $x_{k}:=P_{k}^{\prime \prime} x^{\prime \prime} \in E_{k}$ for each $k \in \mathbb{N}$. Now $\left(P_{k}^{\prime \prime}\right)$ is a $T$-decomposition of $E^{\prime \prime}\left[\sigma\left(E^{\prime \prime}, E^{\prime}\right)\right]$ because $\left(P_{k}\right)$ is shrinking. Therefore, $T\left(x_{k}\right)=$ $T\left(P_{k}^{\prime \prime} x^{\prime \prime}\right)$ is a $\sigma\left(E, E^{\prime}\right)$-Cauchy sequence and the hypothesis of $\beta$-completeness yields the existence of some $x \in E$ such that $P_{k} x=x_{k}$ for every $k \in \mathbb{N}$. Finally we obviously have $x^{\prime \prime}=x$ so that $E$ is semi-reflexive.

This theorem can be dualized to obtain conditions for the barrelledness of a locally convex space with a $T$-decomposition endowed with its Mackey topology. This barrelledness result complements those obtained in [PSV2].

COROLLARY 2.2. Let $E$ be a locally convex space and let $\left(P_{k}\right)$ be a $T$-decomposition of $E$ endowed with its Mackey topology $\mu\left(E, E^{\prime}\right)$. Then the following conditions are equivalent:

(1) $E\left[\mu\left(E, E^{\prime}\right)\right]$ is barrelled.

(2) $\left(P_{k}\right)$ is a $T$-decomposition of $E\left[\beta\left(E, E^{\prime}\right)\right]$, each $E_{k}$ is barrelled and $\left(P_{k}^{\prime}\right)$ is a $\gamma$-complete $T$-decomposition of $E^{\prime}\left[\sigma\left(E^{\prime}, E\right)\right]$.

(3) $\left(P_{k}\right)$ is a $T$-decomposition of $E\left[\beta\left(E, E^{\prime}\right)\right]$, each $E_{k}$ is barrelled and $\left(P_{k}^{\prime}\right)$ is a complete T-decomposition of $E^{\prime}\left[\sigma\left(E^{\prime}, E\right)\right]$.

It is well-known that a locally convex space is reflexive if and only if it is semireflexive and barrelled [Jr, 11.4.2]. A direct application of Theorem 2.1 and Corollary 2.2 gives the following corollaries.

COROLlary 2.3. Let $\left(P_{k}\right)$ be a T-decomposition of a barrelled space $E$. Then the following conditions are equivalent:

(1) $E$ is reflexive.

(2) $\left(P_{k}\right)$ is $\gamma$-complete and shrinking, and each $E_{k}$ is reflexive.

(3) $\left(P_{k}\right)$ is $\beta$-complete and shrinking, and each $E_{k}$ is reflexive. 
COROLLARY 2.4. Let $E$ be a locally convex space and let $\left(P_{k}\right)$ be a $T$-decomposition of $E$ endowed with its Mackey topology $\mu\left(E, E^{\prime}\right)$. Then the following conditions are equivalent:

(1) $E\left[\mu\left(E, E^{\prime}\right)\right]$ is reflexive.

(2) Both $\left(P_{k}\right)$ and $\left(P_{k}^{\prime}\right)$ are $\gamma$-complete and shrinking for the respective weak topologies $\sigma\left(E, E^{\prime}\right)$ and $\sigma\left(E^{\prime}, E\right)$, and each $E_{k}$ is reflexive.

(3) Both $\left(P_{k}\right)$ and $\left(P_{k}^{\prime}\right)$ are complete and shrinking for the respective weak topologies $\sigma\left(E, E^{\prime}\right)$ and $\sigma\left(E^{\prime}, E\right)$, and each $E_{k}$ is reflexive.

\section{Compact sets and Montel spaces}

It is well-known that to deduce that a locally convex space with a basis is a Montel space one must impose some uniform convergence condition on the series representing a given element in terms of the basis elements (see [BMS] for the case of Köthe sequence spaces or [DM2] and [Mi].for the case of Fréchet spaces with Schauder decompositions). The condition we shall consider is a straightforward reformulation of the one introduced by Miñarro [Mi] within the setting of Schauder decompositions of Fréchet spaces, itself being an extension of Mazur's compactness criterion for bases [GW].

DEFINITION. Let $\left(P_{k}\right)$ be a $T$-decömposition of a locally convex space $E$. We say that $\left(P_{k}\right)$ has property $(M)$ if the pointwise convergence to the identity of the sequence of operators $\left(T_{n}\right)$ is uniform in each bounded subset of $E$.

To see that this property is, within our setting, the analogue of Mazur's compactness criterion for bases, we shall make use of a double limit technique. This Double Limit Lemma is certainly well-known for double sequences but we need a reformulation in terms of convergence of nets (one could, just as well, work with filters instead of nets).

Let us recall briefly the terminology and results about nets in locally convex spaces that we shall use (we refer the reader to [Ke, Chapter 2 and Chapter 6] or [Kö, Section 2, Section 6 and Section 15] where the framework is that of topological spaces or uniform spaces).

DEFINITION. A directed set is a pair $(\mathscr{I}, \succ)$ where $\mathscr{I}$ is a set and $\succ$ is a partial order relation in $\mathscr{I}$ such that for every $i_{1}, i_{2} \in \mathscr{I}$ there is $i_{3} \in \mathscr{I}$ such that $i_{3} \succ i_{1}$ and $i_{3} \succ i_{2}$. Let $E$ be a locally convex space. A net in $E$ is a mapping $\left\{x_{i}: i \in \mathscr{I}\right\}$ from $\mathscr{I}$ into $E$ where $(\mathscr{I}, \succ)$ is a directed set; $\mathscr{I}$ is usually called the index set of the net. When the index set is the set $\mathbb{N}$ of all positive integers with its usual ordering, the notion of a net is simply the familiar notion of a sequence. Since there seems to be no possibility of confusion, in what follows we will use the same symbol $\succ$ to denote 
the order relations whenever we have different index sets. A net $\left\{y_{l}: l \in \mathscr{L}\right\}$ is said to be a subnet of $\left\{x_{i}: i \in \mathscr{I}\right\}$ if there is a function $i: l \in \mathscr{L} \rightarrow i(l) \in \mathscr{I}$ such that $y_{l}=x_{i(l)}$ and that for each $i_{0} \in \mathscr{I}$ there is $l_{0} \in \mathscr{L}$ such that if $l \succ l_{0}$, then $i(l) \succ i_{0}$. As it is the case with subsequences, one usually writes $\left\{x_{i(l)}: l \in \mathscr{L}\right\}$ to denote a subnet of a given net $\left\{x_{i}: i \in \mathscr{I}\right\}$. Given two directed sets $(\mathscr{I}, \succ)$ and $(\mathscr{J}, \succ)$, we may define an order relation in $\mathscr{I} \times \mathscr{J}$ by saying that $\left(i_{1}, j_{1}\right) \succ\left(i_{2}, j_{2}\right)$ if $i_{1}>i_{2}$ and $j_{1} \succ j_{2}$. With this order relation the pair $(\mathscr{I} \times \mathscr{J}, \succ)$ is a directed set and a net of the form $\left\{x_{i j}:(i, j) \in \mathscr{I} \times \mathscr{J}\right\}$ is called a double net.

A net $\left\{x_{i}: i \in \mathscr{I}\right\}$ in $E$ is said to be convergent to a point $x \in E$, and we write $x=\lim _{i} x_{i}$, if for every zero-neighborhood $U \in \mathscr{U}(E)$ there is $i_{U} \in \mathscr{I}$ such that $x-x_{i} \in U$ for all $i>i_{U}$; and is said to be a Cauchy net if for every zero-neighborhood $U \in \mathscr{U}(E)$ there is $i_{U} \in \mathscr{I}$ such that $x_{i_{1}}-x_{i_{2}} \in U$ for all $i_{1}, i_{2} \succ i_{U}$. Every convergent net is a Cauchy net and a locally convex space $E$ is said to be complete if every Cauchy net in $E$ is convergent to a point in $E$. Every subnet of a convergent (respectively Cauchy) net is also convergent to the same limit (respectively Cauchy).

LEMMA 3.1 (Double Limit Lemma). Let $E$ be a locally convex space and $\left\{x_{i j}\right.$ : $(i, j) \in \mathscr{I} \times \mathscr{J}\}$ be a double net in $E$ such that for each $i \in \mathscr{I}$ there exists the limit $y_{i}=\lim _{j} x_{i j}$ and for each $j \in \mathscr{J}$ there exists the limit $z_{j}=\lim _{i} x_{i j}$. If the convergence of $\left\{x_{i j}: j \in \mathscr{J}\right\}$ to $y_{i}$ is uniform with respect to $i$ in $\mathscr{I}$, then the three nets $\left\{x_{i j}:(i, j) \in \mathscr{I} \times \mathscr{J}\right\},\left\{y_{i}: i \in \mathscr{I}\right\}$ and $\left\{z_{j}: j \in \mathscr{J}\right\}$ are Cauchy nets. If, in addition, $E$ is complete then the three nets above are convergent to the same limit.

Proof. Let $U \in \mathscr{U}(E)$ be a zero-neighborhood. Since the convergence of $\left\{x_{i j}\right.$ : $j \in \mathscr{J}\}$ to $y_{i}$ is uniform with respect to $i$ in $\mathscr{I}$, there exists $j_{U} \in \mathscr{J}$ such that $q_{U}\left(x_{i j}-y_{i}\right) \leq 1$, for all $j>j_{U}$ and all $i \in \mathscr{I}$. Now $\left\{x_{i j}: i \in \mathscr{I}\right\}$ is a Cauchy net because it converges to $z_{j v}$ by hypothesis, hence there exists $i_{U} \in \mathscr{I}$ such that $q_{U}\left(x_{i_{1} j_{U}}-x_{i_{2} j_{U}}\right) \leq 1$, for all $i_{1}, i_{2} \succ i_{U}$. Therefore, for all $i_{1}, i_{2} \succ i_{U}$ we have

$$
q_{U}\left(y_{i_{1}}-y_{i_{2}}\right) \leq q_{U}\left(y_{i_{1}}-x_{i_{1} j_{U}}\right)+q_{U}\left(x_{i_{1} j_{U}}-x_{i_{2} j_{U}}\right)+q_{U}\left(x_{i_{2} j_{U}}-y_{i_{2}}\right) \leq 3 .
$$

This shows that $\left\{y_{i}: i \in \mathscr{I}\right\}$ is a Cauchy net in $E$. On the other hand, for all $i_{1}, i_{2} \succ i_{U}$ and all $j_{1}, j_{2} \succ j_{U}$ we have

$$
q_{U}\left(x_{i_{1} j_{1}}-x_{i_{2} j_{2}}\right) \leq q_{U}\left(x_{i_{1} j_{1}}-y_{i_{1}}\right)+q_{U}\left(y_{i_{1}}-y_{i_{2}}\right)+q_{U}\left(y_{i_{2}}-x_{i_{2} j_{2}}\right) \leq 5,
$$

hence the double net $\left\{x_{i j}:(i, j) \in \mathscr{I} \times \mathscr{J}\right\}$ is also a Cauchy net in $E$. Finally, use the continuity of the seminorm $q_{U}$ to deduce from the equalities $z_{j_{1}}=\lim _{i} x_{i j_{1}}$ and $z_{j_{2}}=\lim _{i} x_{i j_{2}}$, and the inequality $q_{U}\left(x_{i_{1} j_{1}}-x_{i_{2} j_{2}}\right) \leq 5$, which is true for all $i_{1}, i_{2}>i_{U}$ and $j_{1}, j_{2} \succ j_{U}$, that

$$
q_{U}\left(z_{j_{1}}-z_{j_{2}}\right) \leq 5, \quad \text { for all } j_{1}, j_{2}>j_{U}
$$


So, $\left\{z_{j}: j \in \mathscr{J}\right\}$ is also a Cauchy net in $E$.

If, in addition, $E$ is complete then a straightforward computation shows that the limits of the three Cauchy nets coincide.

LEMMA 3.2. Let $\left(P_{k}\right)$ be a T-decomposition of a locally convex space $E$. Let $D \subset E$ be such that $\lim _{n} T_{n} x=x$ uniformly with respect to $x$ in $D$. Then:

(1) If $P_{k}(D)$ is precompact for each $k \in \mathbb{N}$, then $D$ is precompact.

(2) If $\left(P_{k}\right)$ is complete and $P_{k}(D)$ is relatively compact for each $k \in \mathbb{N}$, then $D$ is relatively compact.

(3) If $\left(P_{k}\right)$ is complete and $D$ is a sequence of the form $D=T\left(x_{k}\right)$ where $x_{k} \in E_{k}$ for each $k \in \mathbb{N}$, then $T\left(x_{k}\right)$ converges in $E$.

PROOF. As it is well-known (see [Jr, 3.5.1], [Ke, Theorem 32 of Chapter 6] or [Kö, Section 5.6]) a set $D$ in a locally convex space is precompact if and only if every net in $D$ has a Cauchy subnet. So, to see (1), consider a net $\left\{z_{r}: r \in \mathscr{R}\right\}$ in $D$ and let us prove that it has a Cauchy subnet. Denote by $\widehat{E}$ the completion of $E$, by $D_{k}$ the set $P_{k}(D)$, and by $\widehat{D}_{k}$ the closure of $D_{k}$ in $\widehat{E}$. Then each $\widehat{D}_{k}$ is a compact subset of $\widehat{E}$ and, consequently, $Z:=\prod_{k=1}^{\infty} \widehat{D}_{k}$ is a compact space for the product topology. Since $\left\{\left(P_{k} z_{r}\right)_{k}: r \in \mathscr{R}\right\}$ is a net in $Z$, there exists a subnet $\left\{\left(P_{k} z_{r(s)}\right)_{k}: s \in \mathscr{S}\right\}$ that converges to some element $\left(x_{k}\right) \in Z$. In particular, for each $n \in \mathbb{N}$ we have $\lim _{s} T_{n} z_{r(s)}=\sum_{k} t_{n k} x_{k}$, the $n$-th term of $T\left(x_{k}\right)$. Since $\lim _{n} T_{n} z_{r(s)}=z_{r(s)}$ is uniform with respect to $s$ in $\mathscr{S}$ by hypothesis, we may apply the Double Limit Lemma, with $\mathbb{N}$ playing the role of $\mathscr{I}$ and $\mathscr{S}$ playing the role of $\mathscr{J}$, to deduce that $\left\{z_{r(s)}: s \in \mathscr{S}\right\}$ is a Cauchy net in $E$ and this concludes the proof that $D$ is precompact.

To see (2), proceed analogously to prove that $T\left(x_{k}\right)$ is a Cauchy sequence noting that, in this case, each $\widehat{D}_{k}$ coincides with the closure of $D_{k}$ in $E_{k}$. Moreover, since $\left(P_{k}\right)$ is a complete $T$-decomposition, what we now obtain is that the Cauchy sequence $T\left(x_{k}\right)$ converges in $E$.

To see (3) denote by $z_{n}$ the $n$-th element of $D=T\left(x_{k}\right)$ and apply the Double Limit Lemma to the double sequence $\left\{T_{p} z_{n}: p, n \in \mathbb{N}\right\}$ : For a fixed $n$ it converges uniformly as $p \rightarrow \infty$ by hypothesis, and for a fixed $p$ it converges to $z_{p}$ as $n \rightarrow \infty$ because $T$ is an $S p_{1}$-matrix and each $x_{k}$ is in $E_{k}$. Therefore, the Double Limit Lemma tells us that $T\left(x_{k}\right)$ is a Cauchy sequence and, since $\left(P_{k}\right)$ is complete, it must converge.

Let us recall at this point that a locally convex space $E$ is said to be semi-Montel if every bounded set of $E$ is relatively compact. The extension to locally convex spaces of the results given by Díaz and Miñarro [DM1, DM2] for Fréchet spaces with Schauder decompositions are contained in the following results.

-THEOREM 3.3. Let $E$ be a locally convex space with a $T$-decomposition $\left(P_{k}\right)$. Consider the conditions 
(1) E is semi-Montel.

(2) $\left(P_{k}\right)$ has property $(M)$ and each $E_{k}$ is semi-Montel.

Then the following hold: If $\left(P_{k}\right)$ is equicontinuous, then (1) implies (2), whereas if $\left(P_{k}\right)$ is complete, then (2) implies (1).

ProOF. Assume that $\left(P_{k}\right)$ is equicontinuous and that (1) holds. Then $\left(T_{n}\right)$ is an equicontinuous sequence that converges pointwise to the identity on $E$. Use that $E$ is semi-Montel and [Kö, Section 39.4.(2)] to deduce that $\left(T_{n}\right)$ converges uniformly on bounded sets. On the other hand, it is obvious that if $E$ is semi-Montel then each $E_{k}$ is semi-Montel.

That (2) plus the completeness of $\left(P_{k}\right)$ imply (1) follows from Lemma 3.2 part (2) above.

REMARK 3.4. (1) Note that a $T$-decomposition of a locally convex space $E$ is shrinking exactly when it has property $(M)$ as a $T$-decomposition of $E\left[\sigma\left(E, E^{\prime}\right)\right]$. In particular, since a locally convex space $E$ is semi-reflexive if and only if $E\left[\sigma\left(E, E^{\prime}\right)\right]$ is semi-Montel, Theorem 3.3 provides an alternative proof of the fact that (3) implies (1) in Theorem 2.1.

(2) Property $(M)$ implies that the decomposition is shrinking. The usual Schauder basis of $\ell^{p}$ (with $1<p<\infty$ ) shows that the converse need not hold.

(3) Property $(M)$ implies that the decomposition is $\gamma$-complete provided that it is complete; see Lemma 3.2 part (3).

(4) The example $\ell^{p}\left[\sigma\left(\ell^{p}, \ell^{q}\right)\right]$ (with $1<p<\infty$ and $p^{-1}+q^{-1}=1$ ) or, more generally, any reflexive infinite-dimensional Banach space endowed with its weak topology, shows that property $(M)$ need not imply equicontinuity. More generally, it can be proved that if $E$ is an infinite-dimensional normed space, then no regular and triangular $T$-decomposition can be an equicontinuous $T$-decomposition of $E\left[\sigma\left(E, E^{\prime}\right)\right]$.

COROLLARY 3.5. Let $\left(P_{k}\right)$ be a T-decomposition of a barrelled and sequentially complete locally convex (in particular, Fréchet) space $E$. Then $E$ is a Montel space if and only if $\left(P_{k}\right)$ has property $(M)$ and each $E_{k}$ is a Montel space.

PROOF. On one hand, barrelledness implies that the decomposition is equicontinuous and sequential completeness implies that the decomposition is complete. On the other hand, every barrelled semi-Montel spaces is a Montel space so the equivalence follows from Theorem 3.3.

COROLLARY 3.6. Let $E$ be a sequentially complete $(D F)$-space with a Toeplitz decomposition $\left(P_{k}\right)$ with respect to a Zeller-Buntinas triangle $T$. Then $E$ is a Montel space if and only if $\left(P_{k}\right)$ has property $(M)$ and each $E_{k}$ is a Montel space. 
PROOF. The conclusion follows from the fact that if $E$ is a sequentially complete $(D F)$-space with a Toeplitz decomposition $\left(P_{k}\right)$ with respect to a Zeller-Buntinas triangle $T$, then $E$ is barrelled if and only if every $E_{k}$ is barrelled [PSV2, Corollary 2 to Theorem 2].

REMARK 3.7. The space $\ell^{\infty}\left[\mu\left(\ell^{\infty}, \ell^{1}\right)\right]$ is a striking example of a non-barrelled, semi-Montel space with a complete Schauder basis that has property $(M)$ because, as is well-known [Kö, Section 22.4.(3)], a bounded subset $C \subset \ell^{1}$ is relatively compact for the weak topology $\sigma\left(\ell^{1}, \ell^{\infty}\right)$ if and only if $\lim _{n} \sup _{x \in C} \sum_{i=n}^{\infty}\left|x_{i}\right|=0$.

\section{Quasi-normable and Schwartz spaces}

Quasi-normable and Schwartz spaces were introduced by Grothendieck in the 1950's. A locally convex space $E$ is said to be quasi-normable if for each $U \in \mathscr{U}(E)$ there exists $V \in \mathscr{U}(E)$ such that for all $\varepsilon>0$ there exists a bounded set $D^{\varepsilon} \subset E$ such that $V \subset D^{\varepsilon}+\varepsilon U$. It is well-known [Jr, 10.7.3] that $E$ is a Schwartz space if and only if it is quasi-normable and all of its bounded subsets are precompact. A subclass of the class of quasi-normable spaces has been defined more recently by Peris [Pr]. A locally convex space $E$ is said to be quasi-normable by operators if for each $U \in \mathscr{U}(E)$ there exists $V \in \mathscr{U}(E)$ such that for all $\varepsilon>0$ there exists a continuous linear mapping $f^{\varepsilon}: E \rightarrow E$ such that $f^{\varepsilon}(V)$ is bounded and $\left(i d_{E}-f^{\varepsilon}\right)(V) \subset \varepsilon U$. This class has much better stability properties than the class of quasi-normable spaces.

As was the case with semi-reflexivity or Montel's property, some conditions must be imposed on the convergence of $\left(T_{n} x\right)$ to $x$ to obtain respective characterizations of quasi-normable (by operator) spaces and Schwartz spaces. For the case of Köthe echelon spaces this was already noted by Grothendieck (see [Gr, BMS, BDe, BD, Is, Mi]). These properties are collected in the following definition.

DEFINITION. Let $\left(P_{k}\right)$ be a $T$-decomposition of a locally convex space $E$. We say that $\left(P_{k}\right)$ has property $(S)$ if for each $U \in \mathscr{U}(E)$ there exists $W \in \mathscr{U}(E)$ such that the convergence of the decomposition in $W$ is uniform with respect to the seminorm induced by $U$, that is, $\lim _{n} \sup \left\{q_{U}\left(x-T_{n} x\right): x \in W\right\}=0$. It is not difficult to see that if $\left(P_{k}\right)$ has property $(S)$ then it also has property $(M)$ and is equicontinuous.

We say that $\left(P_{k}\right)$ has property $(Q N)$ if for each $U \in \mathscr{U}(E)$ there exists $W \in \mathscr{U}(E)$ such that for all $\varepsilon>0$ and $k \in \mathbb{N}$ there exists a bounded set $D_{k}^{\varepsilon} \subset E_{k}$ such that

$$
\left(W \cap E_{k}\right) \subset D_{k}^{\varepsilon}+\varepsilon\left(U \cap E_{k}\right) .
$$

Property $(Q N)$ means that all the steps $E_{k}$ are quasi-normable in a uniform way, that is, given the zero-neighborhoods $U \cap E_{k}$, the corresponding zero-neighborhoods 
$W_{k}$ that would ensure the quasi-normability of each $E_{k}$ are all of the form $W_{k}=$ $W \cap E_{k}$ for some fixed zero-neighborhood $W \in \mathscr{U}(E)$ that depends only on $U$. This condition $(Q N)$ is weaker than Miñarro's condition ( $q n)$ [Mi, 4.12] defined for Schauder decompositions on Fréchet spaces.

We say that $\left(P_{k}\right)$ has property $(Q N O)$ if for each $U \in \mathscr{U}(E)$ there exists $W \in$ $\mathscr{U}(E)$ such that for all $\varepsilon>0$ and $k \in \mathbb{N}$ there exists a continuous linear mapping $f_{k}^{\varepsilon}: E_{k} \rightarrow E_{k}$ such that $f_{k}^{\varepsilon}\left(W \cap E_{k}\right)$ is bounded and

$$
\left(i d_{E_{k}}-f_{k}^{\varepsilon}\right)\left(W \cap E_{k}\right) \subset \varepsilon\left(U \cap E_{k}\right) .
$$

Again, property $(Q N O)$ means that all the steps $E_{k}$ are quasi-normable by operators in a uniform way. Of course, property $(Q N O)$ implies property $(Q N)$.

Property $(S)$ by itself does not imply property $(Q N)$, even for Schauder decompositions (see the Remark following Theorem 4.2 below). However, if $\left(P_{k}\right)$ is a finite-dimensional $T$-decomposition, then both $(Q N)$ and $(Q N O)$ hold automatically. To see this consider $U \in \mathscr{U}(E)$ and take $W=U$. Then $U \cap E_{k}$ is an absolutely convex set in the finite-dimensional space $E_{k}$, so we may apply [Jr, 10.6.2] to deduce that given $\varepsilon>0$ there exists a finite number of elements $x_{1}^{\varepsilon}, x_{2}^{\varepsilon}, \ldots, x_{m_{k}}^{\varepsilon} \in E_{k}$, with $m_{k}=\operatorname{dim}\left(E_{k}\right)$ such that

$$
U \cap E_{k} \subset \operatorname{acx}\left(\left\{x_{1}^{\varepsilon}, x_{2}^{\varepsilon}, \ldots, \overline{x_{m_{k}}^{\varepsilon}}\right\}\right)+\varepsilon\left(U \cap E_{k}\right) .
$$

Here $\operatorname{acx}(B)$ denotes the closed, absolutely convex hull of a set $B$. Now, for each $k \in \mathbb{N}$, define the set $D_{k}:=\operatorname{acx}\left(\left\{x_{1}^{\varepsilon}, x_{2}^{\varepsilon}, \ldots, x_{m_{k}}^{\varepsilon}\right\}\right)$ to prove that $(Q N)$ holds, and take any linear mapping $f_{k}^{\varepsilon}$ such that $f_{k}^{\varepsilon}\left(U \cap E_{k}\right) \subset \operatorname{acx}\left(\left\{x_{1}^{\varepsilon}, x_{2}^{\varepsilon}, \ldots, x_{m_{k}}^{\varepsilon}\right\}\right)$ to prove that $(Q N O)$ holds.

The theorems in this section are formulated for the case when $T$ is a Zeller-Buntinas triangle $T$ that can be factorized as $T=A \Sigma$ for some regular triangle $A$. Recall that, in this case, $\left|t_{n k}\right| \leq\|A\|$ for all $n, k \in \mathbb{N}$, where $\|A\|$ is the row-norm of $A$. We use this notation throughout the section.

LEMMA 4.1. Let $T$ be a Zeller-Buntinas triangle with equicontinuity constant $b_{T}$. Let $E$ be a locally convex space with an equicontinuous $T$-decomposition $\left(P_{k}\right)$. Then there is a basis $\mathscr{U}_{T}(E)$ of absolutely convex and closed zero-neighborhoods in $E$ such that

$$
q_{U}\left(T_{n} x\right) \leq b_{T} q_{U}(x) \quad \text { for every } x \in E, n \in \mathbb{N} \text { and } U \in \mathscr{U}_{T}(E) \text {. }
$$

PROOF. Fix any basis $\mathscr{U}(E)$ of absolutely convex and closed zero-neighborhoods defining the topology of $E$ and for every $U \in \mathscr{U}(E)$ define a new seminorm on $E$ by

$$
q_{U}^{T}(x):=\sup \left\{q_{U}\left(T_{n} x\right): n \in \mathbb{N}\right\} .
$$


A straightforward computation, using the equicontinuity of the operators $\left(T_{n}\right)$ and the fact that $\left(T_{n} x\right)$ converges to $x$ for all $x \in E$, shows that the family of seminorms $\left\{q_{U}^{T}: U \in \mathscr{U}(E)\right\}$ also defines the topology of $E$. Fix a seminorm $q_{U}^{T}$ of this type, $x \in E$ and $n \in \mathbb{N}$. Let $U^{\circ}$ be the polar of $U$ in $E^{\prime}$. Given $x^{\prime} \in U^{\circ}$, the scalar sequence $\left(\left\langle P_{k} x, x^{\prime}\right\rangle\right)$ is in $c_{T}$ so, by the definition of \|\|$_{T}$, we may write

$$
q_{U}^{T}(x)=\sup \left\{\left|\left\langle T_{n} x, x^{\prime}\right\rangle\right|: n \in \mathbb{N}, x^{\prime} \in U^{\circ}\right\}=\sup \left\{\left\|\left(\left\langle P_{k} x, x^{\prime}\right\rangle\right)\right\|_{T}: x^{\prime} \in U^{\circ}\right\} .
$$

To estimate $q_{U}^{T}\left(T_{n} x\right)$ note that the orthogonality of the projections $\left(P_{k}\right)$ implies, for every $k \in \mathbb{N}$ and $x^{\prime} \in U^{\circ}$, that $\left(\left\langle P_{k} T_{n} x, x^{\prime}\right\rangle\right)=\tau_{n}\left(\left\langle P_{k} x, x^{\prime}\right\rangle\right)$. Therefore

$$
\begin{aligned}
q_{U}^{T}\left(T_{n} x\right) & =\sup \left\{\left\|\left(\left\langle P_{k} T_{n} x, x^{\prime}\right\rangle\right)\right\|_{T}: x^{\prime} \in U^{\circ}\right\}=\sup \left\{\left\|\tau_{n}\left(\left\langle P_{k} x, x^{\prime}\right\rangle\right)\right\|_{T}: x^{\prime} \in U^{\circ}\right\} \\
& \leq b_{T} \sup \left\{\left\|\left(\left\langle P_{k} x, x^{\prime}\right\rangle\right)\right\|_{T}: x^{\prime} \in U^{\circ}\right\}=b_{T} q_{U}^{T}(x)
\end{aligned}
$$

for all $n \in \mathbb{N}$ and $x \in E$. Now take $\mathscr{U}_{T}(E)$ to be the closed unit balls determined by the family of seminorms $\left\{q_{U}^{T}: U \in \mathscr{U} .(E)\right\}$.

THEOREM 4.2. Let $T$ be a Zeller-Buntinas triangle that can be factorized as $T=A \Sigma$ for some regular triangle $A$. Let $E$ be a locally convex space with a $T$-decomposition $\left(P_{k}\right)$ that has property $(S)$. Then

(1) $E$ is quasi-normable if and only if $\left(P_{k}\right)$ has property $(Q N)$.

(2) $E$ is quasi-normable by operators if and only if $\left(P_{k}\right)$ has property $(Q N O)$.

PROOF. Since property $(S)$ implies that the $T$-decomposition is equicontinuous, we may use the previous lemma to restrict ourselves to the case when the basis of zero-neighborhoods is the family $\mathscr{U}_{T}(E)$. In what follows, we denote the triangle $T^{-1}$ by $\left[s_{k n}\right]$ and, for each $k \in \mathbb{N}$, define $\alpha_{k}:=\sum_{n=1}^{k}\left|s_{k n}\right|$.

The direct implications are not as straightforward as might seem at first glance. For, in general, we do not have $(A+B) \cap C \subset(A \cap C)+(B \cap C)$, even if $A, B$ and $C$ are convex and closed sets of a locally convex space $E$. For instance, take $A$ to be the unit interval in the $X$-axis of $\mathbb{R}^{2}, B$ the unit interval in the $Y$-axis and $C$ the segment joining the origin and the point $(1,1)$. So we need to carefully check all the details. To prove the direct implication in (1) let $U \in \mathscr{U}_{T}(E)$ be given. Since $E$ is quasi-normable, there is $W \in \mathscr{U}_{T}(E)$ such that for all $\delta>0$ there is a bounded set $D \subset E$ (depending on $U, W$ and $\delta$ ) such that $W \subset D+\delta U$. Let us see that $W$ is as required by property $(Q N)$. Given $\varepsilon>0$ and $k \in \mathbb{N}$, let $\delta=\varepsilon /\left(b_{T} \alpha_{k}\right)$ and, with the bounded set $D$ corresponding to $U, W$ and this value of $\delta$, define $D_{k}:=P_{k}(D)$ which, obviously, is a bounded subset of $E_{k}$. We have to prove that

$$
\left(W \cap E_{k}\right) \subset D_{k}+\varepsilon\left(U \cap E_{k}\right) \text {. }
$$


Since $P_{k}$ is linear, it is clear that

$$
\left(W \cap E_{k}\right) \subset P_{k}(W) \subset P_{k}(D)+\delta P_{k}(U)=D_{k}+\delta P_{k}(U),
$$

so it remains to prove that $\delta P_{k}(U) \subset \varepsilon\left(U \cap E_{k}\right)$. Now, if $x \in U$ then, using the property established in Lemma 4.1,

$$
q_{U}\left(P_{k} x\right)=q_{U}\left(\sum_{n} s_{k n} T_{n} x\right) \leq \alpha_{k} q_{U}\left(T_{n} x\right) \leq \alpha_{k} b_{T} q_{U}(x),
$$

so that $\delta P_{k}(x) \in \delta \alpha_{k} b_{T}\left(U \cap E_{k}\right)=\varepsilon\left(U \cap E_{k}\right)$.

To prove the direct implication in (2), proceed analogously making suitable changes; in particular, take $f_{k}:=P_{k}\left(f \mid E_{k}\right)$, the projection into $E_{k}$ of the restriction to $E_{k}$ of the existing $f$.

To prove the reverse implication in (1), let $U \in \mathscr{U}_{T}(E)$ be given. Then we may assume that a single $W \in \mathscr{U}_{T}(E)$ works for both $(S)$ and $(Q N)$. Then for each $\varepsilon>0$ and $k \in \mathbb{N}$ there exists $n_{0} \in \mathbb{N}$ and a bounded set $D_{k} \subset E_{k}$ (depending on $U, W, n_{0}$ and $\varepsilon$ ) such that

$$
\begin{gathered}
q_{U}\left(x-T_{n_{0}} x\right) \leq \varepsilon q_{W}(x) \quad \text { for all } x \in E \text { and } \\
W \cap E_{k} \subset D_{k}+\frac{\varepsilon}{\|A\| \alpha_{k} 2^{k}}\left(U \cap E_{k}\right) .
\end{gathered}
$$

Since, as pointed out above, property $(S)$ implies the decomposition is equicontinuous, there exists a zero-neighborhood $V \subset W$ such that $q_{W}\left(T_{n} x\right) \leq q_{V}(x)$ for all $n \in \mathbb{N}$. Using that $P_{k}$ is the $k$-th term of the sequence $T^{-1}\left(T_{n}\right)$, for each $k \in \mathbb{N}$ and $x \in E$ we have

$$
q_{W}\left(P_{k} x\right)=q_{W}\left(\sum_{n=1}^{k} s_{k n} T_{n} x\right) \leq \sum_{n=1}^{k}\left|s_{k n}\right| q_{W}\left(T_{n} x\right) \leq \alpha_{k} q_{V}(x) .
$$

We claim that the bounded set $D:=\sum_{k=1}^{n_{0}} t_{n_{0}} \alpha_{k} D_{k}$ satisfies $V \subset D+2 \varepsilon U$. Indeed, given $x \in V$ we have $x-T_{n_{0}} x \in \varepsilon U$ by inequality (i), so it is enough to show that $T_{n_{0}} x \in D+\varepsilon U$. It follows from (iii) that $\alpha_{k}^{-1} P_{k} x \in W \cap E_{k}$. Now, use (ii) to obtain that for each $k=1,2, \ldots, n_{0}$ there exist $d_{k} \in D_{k}$ and $x_{k} \in\left(U \cap E_{k}\right)$ such that

$$
\alpha_{k}^{-1} P_{k} x=d_{k}+\frac{\varepsilon}{\|A\| \alpha_{k} 2^{k}} x_{k} .
$$

Accordingly,

$$
\begin{aligned}
T_{n_{0}} x & =\sum_{k=1}^{n_{0}} t_{n_{0} k} P_{k} x=\sum_{k=1}^{n_{0}} t_{n_{0} k} \alpha_{k}\left(d_{k}+\frac{\varepsilon}{\|A\| \alpha_{k} 2^{k}} x_{k}\right) \\
& =\sum_{k=1}^{n_{0}} t_{n_{0} k} \alpha_{k} d_{k}+\varepsilon \sum_{k=1}^{n_{0}} \frac{t_{n_{0} k}}{\|A\| 2^{k}} x_{k} .
\end{aligned}
$$


Finally, note that the first sum is in $D$ and the second sum is in $\varepsilon U$ because $\left|t_{n k}\right| \leq\|A\|$ for all $n, k \in \mathbb{N}$ and $U$ is absolutely convex.

To prove the reverse implication in (2), proceed analogously making suitable changes; in particular, take $f:=\sum_{k=1}^{n_{0}} t_{n_{0} k} f_{k} P_{k}$.

REMARK 4.3. Grothendieck conjectured [Gr, Chapter II, p. 102] that condition $(S)$ by itself would imply that a (double index) Köthe echelon space is quasi-normable provided that is has a Schauder decomposition (other than the natural one) with property $(S)$ (reformulated in terms of the defining Köthe matrix). However, this conjecture turned out to be false [BMS, Example 3.11] even if all the steps of the decomposition are normable. This lead to the introduction of property $(Q N)$ (again reformulated in terms of the defining Köthe matrix).

THEOREM 4.4. Let $T$ be a Zeller-Buntinas triangle that can be factorized as $T=A \Sigma$ for some regular triangle $A$. Let $E$ be a locally convex space with a $T$-decomposition $\left(P_{k}\right)$. Then the following hold.

(1) If $E$ is a Schwartz space and $\left(P_{k}\right)$ is equicontinuous, then $\left(P_{k}\right)$ has properties $(S)$ and $(Q N)$.

(2) If $\left(P_{k}\right)$ has properties $(S)$ and $(Q N)$ and the bounded subsets of each $E_{k}$ are precompact, then $E$ is a Schwartz space.

ProOF. (1) (Note, for later use, that the hypothesis that $T$ is a Zeller-Buntinas triangle of the form $A \Sigma$ is not used in this part to prove that $\left(P_{k}\right)$ has property $(S)$; we only use that $T$ is row-finite). Since $E$ is a Schwartz space, it is quasi-normable and, by the previous theorem, it follows that $\left(P_{k}\right)$ has property $(Q N)$. Let us now see that $\left(P_{k}\right)$ also has property $(S)$. Given $U \in \mathscr{U}(E)$, the equicontinuity of $\left(P_{k}\right)$ ensures that there exists $W \in \mathscr{U}(E)$ such that $W \subset U$ and $q_{U}\left(T_{n} x\right) \leq q_{W}(x)$ for all $x \in E$ and $n \in \mathbb{N}$. Use again that $E$ is quasi-normable to ensure that there exists $V \in \mathscr{U}(E)$ such that for all $\varepsilon>0$ there exists a bounded set $D^{\varepsilon} \subset E$ with $V \subset D^{\varepsilon}+\varepsilon W$. We shall prove that $\lim _{n} \sup \left\{q_{U}\left(x-T_{n} x\right): x \in V\right\}=0$. Fix $\delta>0$ and consider the corresponding bounded set $D^{\delta}$. This set $D^{\delta}$ is precompact because $E$ is a Schwartz space. Therefore, there exists a finite collection $x_{1}, x_{2}, \ldots, x_{m} \in E$ such that $D^{\delta} \subset \bigcup_{j=1}^{m}\left(x_{j}+\delta W\right)$ and we may take $n_{0} \in \mathbb{N}$ such that $q_{V}\left(x_{j}-T_{n} x_{j}\right) \leq \delta$ for all $j=1,2, \ldots, m$ and $n \geq n_{0}$. Now, given $z \in V$, since $V \subset D^{\delta}+\delta W \subset \bigcup_{j=1}^{m}\left(x_{j}+2 \delta W\right)$, there must exist some $j \in\{1,2, \ldots, m\}$ such that $z-x_{j} \in 2 \delta W$. Therefore, for all $n \geq n_{0}$ we have

$$
\begin{aligned}
q_{U}\left(z-T_{n} z\right) & \leq q_{U}\left(z-x_{j}\right)+q_{U}\left(x_{j}-T_{n} x_{j}\right)+q_{U}\left(T_{n} x_{j}-T_{n} z\right) \\
& \leq q_{W}\left(z-x_{j}\right)+\delta+q_{W}\left(z-x_{j}\right) \leq 5 \delta
\end{aligned}
$$

where we have used the facts that $W \subset U$ and $q_{U}\left(T_{n} x\right) \leq q_{W}(x)$ for all $x \in E$ and $n \in \mathbb{N}$. Since $z$ is arbitrary in $V$, this shows that $\left(P_{k}\right)$ has property $(S)$. 
(2) By the previous theorem $E$ is quasi-normable. Since property $(S)$ implies property $(M)$, it follows from Lemma 3.2.(1) and the hypothesis that the bounded subsets of each $E_{k}$ are precompact that the bounded subsets of $E$ are also precompact. Therefore, $E$ is a Schwartz space.

A careful reading of the above proofs shows that the hypothesis that $T$ is a ZellerBuntinas matrix is used only to deduce that if $E$ is quasi-normable (respectively by operators), then $E$ has property $(Q N)$ (respectively $(Q N O)$ ). Using this remark and the fact that finite-dimensional $T$-decompositions always have property $(Q N)$ we obtain the following corollaries.

COROLLARY 4.5. Let $T$ be a triangle that can be factorized as $T=A \Sigma$ for some regular triangle $A$. Let $E$ be a locally convex space with a finite-dimensional and equicontinuous $T$-decomposition $\left(P_{k}\right)$. Then $E$ is a Schwartz space if and only if $\left(P_{k}\right)$ has property $(S)$.

COROLLARY 4.6. Let $T$ be a triangle that can be factorized as $T=A \Sigma$ for some regular triangle $A$. Let $E$ be a Fréchet space with a finite-dimensional $T$ decomposition $\left(P_{k}\right)$. Then $E$ is a Schwartz space if and only if $\left(P_{k}\right)$ has property $(S)$.

\section{Projective tensor products}

In this section we study the stability of some structural properties in projective tensor products. The general problem is the following: given two locally convex spaces $E$ and $F$ having a certain property $(P)$ to find out whether the projective tensor product $E \widehat{\otimes}_{\pi} F$ inherits that property $(P)$. The first positive answers were obtained by Grothendieck [Gr] for products of Fréchet spaces and the properties of quasi-normability and being a Schwartz space. Since then, many papers on this topic have been published (see [BMS, BDe, BD, BDT, Jr] or [PeB]). The answer is not always positive for other properties (such as distinguishedness, Montel, reflexivity). So, the question arises: Under what conditions does the projective tensor product inherit the properties of its factors? The main difficulty seems to be the fact that one cannot always locate the bounded sets of the projective tensor product via tensor products of bounded subsets of each factor (see [BDT, Pr, T] and references therein). As it turns out, most of the questions have a positive answer when every bounded subset of the tensor product is contained in the closed hull of the tensor product of two bounded sets of each factor. When this occurs the pair $(E, F)$ is said to have property $(B B)$. Our starting point is, again, the work of Díaz and Miñarro [DM1, DM2]. In these two papers, they assume that one of the Fréchet factors has a suitably 'good' Schauder decomposition that enables them to decompose the projective tensor product 
and to link the properties of the factors. In this section we present some results of this type, offering somewhat simplified proofs, valid within the setting of Toeplitz decompositions.

Throughout this section $E$ and $F$ stand for a pair of locally convex spaces and $E \otimes_{\pi} F$ will denote their projective tensor product. The projective tensor product topology is defined by means of the seminorms

$$
\pi_{U V}(z):=\inf \left\{\sum_{i=1}^{n} q_{U}\left(x_{i}\right) q_{V}\left(y_{i}\right): z=\sum_{i=1}^{n} x_{i} \otimes y_{i}\right\}
$$

where $U$ runs through $\mathscr{U}(E), V$ runs through $\mathscr{U}(F)$ and the infimum in taken over all possible finite representations of $z \in E \otimes F$. As usual, the completion of $E \otimes_{\pi} F$ will be denoted by $E \widehat{\otimes}_{\pi} F$. We start by studying how properties of a $T$-decomposition of one factor lift to a natural $T$-decomposition induced on the product.

PROPOSITION 5.1. Let $\left(P_{k}\right)$ be an equicontinuous $T$-decomposition of $E$. Then the following hold.

(1) $\left(P_{k} \otimes i d_{F}\right)$ is an equicontinuous $T$-decomposition of $E \widehat{\otimes}_{\pi} F$ with $E_{k} \widehat{\otimes}_{\pi} F$ as step subspaces.

(2) If $(E, F)$ has property $(B B)$ and $\left(P_{k}\right)$ has property $(M)$, then $\left(P_{k} \otimes i d_{F}\right)$ has property $(M)$.

(3) If $\left(P_{k}\right)$ has property $(S)$, then $\left(P_{k} \otimes i d_{F}\right)$ has property $(S)$.

PROOF. (1) The proof is a straightforward computation for elements in $E \otimes F$. Then use equicontinuity to deduce (1). To prove (2) assume that $B \subset E$ and $C \subset F$ are bounded sets. Given $z \in \operatorname{acx}(B \otimes C)$ write it as $z=\sum_{i=1}^{m} \lambda_{i} x_{i} \otimes y_{i}$ where $\left(x_{i}, y_{i}\right) \in B \times C$ for each $i=1,2, \ldots, m$ and $\sum_{i=1}^{m}\left|\lambda_{i}\right| \leq 1$. Given $U \in \mathscr{U}(E)$ and $V \in \mathscr{U}(F)$ we have

$$
\begin{aligned}
\pi_{U V}\left(z-\left(T_{n} \otimes i d_{F}\right) z\right) & =\pi_{U V}\left(\sum_{i=1}^{m} \lambda_{i}\left(x_{i}-T_{n} x_{i}\right) \otimes y_{i}\right) \\
& \leq \sum_{i=1}^{m}\left|\lambda_{i}\right| q_{U}\left(x_{i}-T_{n} x_{i}\right) q_{V}\left(y_{i}\right) \\
& \leq\left[\sup _{x \in B} q_{U}\left(x-T_{n} x\right)\right]\left[\sup _{y \in C} q_{V}(y)\right] .
\end{aligned}
$$

The equicontinuity of the $T$-decomposition implies that the convergence is uniform on the closure of acx $(B \otimes C)$ and property $(B B)$ implies that it is uniform over every bounded set of $E \widehat{\otimes}_{\pi} F$. This proves (2).

The proof of (3) is analogous. 
We are now ready to extend to the setting of Toeplitz decompositions some of the results obtained by Díaz and Miñarro for tensor products of Fréchet spaces [DM1, DM2].

THEOREM 5.2. Let $\left(P_{k}\right)$ be a finite-dimensional T-decomposition of $E$. Assume that either the pair $(E, F)$ has property $(B B)$ and $\left(P_{k}\right)$ is equicontinuous and has property $(M)$, or that $\left(P_{k}\right)$ has property $(S)$. Then the following hold.

(1) If $F$ is semi-reflexive, then $E \widehat{\bigotimes}_{\pi} F$ has the same property.

(2) If $F$ is semi-Montel, then $E \widehat{\otimes}_{\pi} F$ has the same property.

PrOOF. By applying Lemma 3.2, Remark 3.4 and Proposition 5.1 we obtain that, in either case, $\left(P_{k} \otimes i d_{F}\right)$ is an equicontinuous, shrinking and $\gamma$-complete $T$ decomposition of $E \widehat{\otimes}_{\pi} F$ having property $(M)$. On the other hand, since each $E_{k}$ is finite-dimensional, each step $E_{k} \widehat{\otimes}_{\pi} F$ is isomorphic to a finite product of copies of $F$. In particular, each $E_{k} \widehat{\otimes}_{\pi} F$ is semi-reflexive or semi-Montel whenever $F$ is. Finally, apply Theorem 2.1 and Theorem 3.3.

COROLLARY 5.3. Let $\left(P_{k}\right)$ be a finite-dimensional T-decomposition of a Fréchet space $E$. Assume that either the pair $(E, F)$ has property $(B B)$ and $E$ is a Montel space, or that $E$ is a Schwartz space. Then the following hold.

(1) If $F$ is reflexive, then $E \widehat{\otimes}_{\pi} F$ is reflexive.

(2) If $F$ is Montel, then $E \widehat{\otimes}_{\pi} F$ is Montel.

(3) If $F$ is distinguished and $T$ is a Zeller-Buntinas triangle, then $E \widehat{\otimes}_{\pi} F$ is distinguished.

Proof. If $E$ is Montel, then $\left(P_{k}\right)$ has property $(M)$, by Corollary 3.5 , and is equicontinuous because $E$ is barrelled. If $E$ is Schwartz, then $\left(P_{k}\right)$ has property $(S)$ as was pointed out in the proof of Theorem 4.3.(1). Therefore, (1) and (2) follow from Theorem 5.2. To prove (3) note again, since each $E_{k}$ is finite-dimensional, that each step $E_{k} \widehat{\otimes}_{\pi} F$ is isomorphic to a finite product of copies of $F$. In particular, each $E_{k} \widehat{\otimes}_{\pi} F$ is distinguished whenever $F$ is. Since $\left(P_{k}\right)$ is shrinking, the conclusion follows from [PSV2, Corollary 4].

EXAMPLES 5.4. (1) Taskinen [T] exhibited a Fréchet-Montel space $E$ having a finite-dimensional and unconditional Schauder decomposition having property $(M)$ but such that $E \widehat{\otimes}_{\pi} E$ is not a Montel space. This shows that property $(B B)$ cannot be suppressed in general to lift the property $(M)$ from $\left(P_{k}\right)$ to $\left(P_{k} \otimes i d_{F}\right)$ in Theorem 5.2.(2).

(2) Let $\lambda$ be a Fréchet $K$-space of sequences containing $\varphi$ and having property $T-A K$. As mentioned in the introduction, the coordinate basis is a onedimensional Toeplitz decomposition with respect to the row-finite and $S p_{1}$ matrix 
$T$. Let $\left\{a^{(n)}: n \geq 1\right\}$ be an echelon system on $\lambda$, that is, each step sequence $a^{(n)}:=\left(a_{k}^{(n)}\right)$ is such that every $a_{k}^{(n)} \neq 0$ and $\left(a^{(n)} / a^{(n+1)}\right) x \in \lambda$ for all $n \in \mathbb{N}$ and $x \in \lambda$. Then the injections $\left(1 / a^{(n+1)}\right) \lambda \hookrightarrow\left(1 / a^{(n)}\right) \lambda$ are continuous and the echelon space $E:=\bigcap_{n}\left(1 / a^{(n)}\right) \lambda$ endowed with the projective limit topology is a Fréchet $K$-space (see [FP] and references therein). If $\left(q_{j}\right)$ is a sequence of seminorms generating the topology of $\lambda$, then the topology of $E$ is generated by the seminorms $q_{j n}(x):=q_{j}\left(a^{(n)} x\right)$ as $j, n$ run through $\mathbb{N}$. Let us show that the sequence of projections $\left(\pi_{k}\right)$ (for the notation see Example 1.1) is a $T$-decomposition of $E$. It is easy to check that for every $m \in \mathbb{N}$ we have

$$
\tau_{m}\left(a^{(n)} x\right)=\left(t_{m k} a_{k}^{(n)} x_{k}\right)_{k}=a^{(n)}\left(\tau_{m} x\right)
$$

Now use that $\lambda$ has property $T-A K$ to deduce that

$$
q_{j n}\left(x-\tau_{m} x\right)=q_{j}\left(a^{(n)} x-a^{(n)}\left(\tau_{m} x\right)\right)=q_{j}\left(a^{(n)} x-\tau_{m}\left(a^{(n)} x\right)\right) \underset{m \rightarrow \infty}{\longrightarrow} 0 .
$$

Let $S(\lambda)$ be the closure of $\varphi$ in the multiplier space from $\lambda$ into $\lambda$, seen as the space of all continuous diagonal matrix mappings from $\lambda$ into itself endowed with the topology of uniform convergence on the bounded subsets of $\lambda$. It was proved in [FP] that if for each $n \in N$ there exists $j>n$ such that $\left(a^{(n)} / a^{(j)}\right) \in S(\lambda)$, then $E$ is a Montel space. If, additionally, $\lambda$ is a Banach space, then this condition is equivalent to $E$ being a Schwartz space. This gives us examples of spaces $E$ without natural Schauder basis to which Corollary 5.3 can be applied.

\section{Application to Cesàro bases}

Next in importance to Schauder bases are Cesàro bases. If Schauder bases are interpreted as one-dimensional decompositions with respect to the matrix $\Sigma$ of ordinary summability, then Cesàro bases are one-dimensional decompositions with respect to the triangular matrix of Cesàro summability

$$
C_{1}:=\left[\begin{array}{ccccc}
1 & & & & \\
1 & 1 / 2 & & & \\
1 & 2 / 3 & 1 / 3 & & \\
1 & 3 / 4 & 2 / 4 & 1 / 4 & \\
\vdots & \vdots & \vdots & \vdots & \ddots
\end{array}\right]
$$


Of course, $C_{1}$ is obtained as the product $A_{1} \Sigma$ where $A_{1}$ is the regular triangle

$$
A_{1}:=\left[\begin{array}{ccccc}
1 & & & & \\
1 / 2 & 1 / 2 & & & \\
1 / 3 & 1 / 3 & 1 / 3 & & \\
1 / 4 & 1 / 4 & 1 / 4 & 1 / 4 & \\
\vdots & \vdots & \vdots & \vdots & \ddots
\end{array}\right]
$$

As pointed out above, $C_{1}$ is the first example of a Zeller-Buntinas triangle different from $\Sigma$. The Cesàro duality between sequence spaces, in which $c_{C_{1}}$ plays the role that $\ell^{1}$ plays in Köthe duality, was studied by Florencio [F1, F2, F3] and Florencio and Pérez Carreras [FPe1, FPe2]. We think that it is valuable to rewrite some of the above results within this context.

THEOREM 6.1. Let $E$ and $F$ be locally convex spaces and assume that $E$ has a Cesàro basis.

(1) The following conditions are equivalent.

(1.1) E is semi-reflexive.

(1.2) The basis is $\gamma$-complete and shrinking.

(1.3) The basis is $\beta$-complete and shrinking.

(2) If $E$ is a Fréchet space, then $E$ is a Montel space if and only if the basis has property $(M)$.

(3) If the basis is equicontinuous, then $E$ is a Schwartz space if and only if the basis has property $(S)$.

(4) If the basis is equicontinuous and has property $(M)$, the pair $(E, F)$ has property $(B B)$ and $F$ is semi-reflexive or semi-Montel, then $E \widehat{\otimes}_{\pi} F$ has the same property as $F$.

(5) If the basis has property $(S)$ and $F$ is semi-reflexive or semi-Montel, then $E \widehat{\otimes}_{\pi} F$ has the same property as $F$.

(6) If $E$ is a Fréchet-Montel space, the pair $(E, F)$ has property $(B B)$ and $F$ is distinguished, then $E \widehat{\otimes}_{\pi} F$ has the same property as $F$.

\section{Acknowledgements}

We thank J. C. Díaz Alcaide (Córdoba, Spain) for his valuable comments and remarks. We also thank the referee, whose remarks have greatly improved the readability of the paper. 


\section{References}

[BMS] K. D. Bierstedt, R. Meise and W. H. Summers, 'Köthe sets and Köthe sequence spaces', in: Functional analysis, holomorphy and approximation theory, Math. Studies, vol. 71 (Elsevier/North-Holland, Amsterdam, 1982) pp. 27-91.

[BDe] J. Bonet and A. Defant, 'Projective tensor products of distinguished Fréchet spaces', Proc. Roy. Irish Acad. 85A (1985), 193-199.

[BD] J. Bonet and J. C. Díaz, 'The problem of topologies of Grothendieck and the class of Fréchet $T$-spaces', Math. Nachr. 150 (1991), 109-118.

[BDT] J. Bonet, J. C. Díaz and J. Taskinen, 'Tensor stable Fréchet and (DF)-spaces', Collect. Math. 42 (1991), 199-236.

[Bu1] M. Buntinas, 'On sectionally dense summability fields', Math. Z. 132 (1973), 141-149.

[Bu2] — 'On Toeplitz sections in sequence spaces', Math. Proc. Cambridge Philos. Soc. 78 (1975), 451-460.

[DM1] J. C. Díaz and M. A. Miñarro, 'Distinguished Fréchet spaces and projective tensor product', Doğa Mat. 14 (1990), 191-208.

[DM2] ' 'On Fréchet Montel spaces and their projective tensor product', Math. Proc. Cambridge Philos. Soc. 113 (1993), 335-341.

[F1] M. Florencio, 'Sobre C-dualidad y espacios C-perfectos', Rev. Real Acad. Cienc. Exact. Fís. Natur. Madrid 75 (1981), 1221-1234 (in Spanish).

[F2] - 'Sobre la propiedad $A K$ y $A K-C$ en algunos espacios de sucesiones', Rev. Real Acad. Cienc. Exact. Fís. Natur. Madrid 76 (1982), 575-584 (in Spanish).

[F3] _ 'Una nota sobre espacios escalonados generales', Rev. Real Acad. Cienc. Exact. Fís. Natur. Madrid 77 (1983), 221-226 (in Spanish).

[FPe1] M. Florencio and P. Pérez Carrerás, 'Sobre sumabilidad Cesàro en el espacio CS (I)', Rev. Real Acad. Cienc. Exact. Fís. Natur. Madrid 75 (1981), 1185-1197 (in Spanish).

[FPe2] — 'Sobre sumabilidad Cesàro en el espacio CS (II)', Rev. Real Acad. Cienc. Exact. Fís. Natur. Madrid 76 (1982), 531-546 (in Spanish).

[FP] M. Florencio and P. J. Paúl, 'Some results on diagonal maps', Bull. Soc. Roy. Sci. Liège 55 (1986), 569-580.

[GW] S. Goes and R. Welland, 'Compactness criteria for Köthe spaces', Math. Ann. 188 (1970), 251-269.

[Gr] A. Grothendieck, Produits tensoriels topologiques et espaces nucléaires, Mem. Amer. Math. Soc. 16 (Amer. Math. Soc., Providence, 1955).

[Is] J. M. Isidro, 'Quasinormability of some spaces of holomorphic mappings', Rev. Mat. Univ. Complutense Madrid 3 (1990), 57-123.

[Ja] R. C. James, 'Bases and reflexivity of Banach spaces', Ann. of Math. 52 (1950), 518-527.

[Jr] H. Jarchow, Locally convex spaces (B. G. Teubner, Stuttgart, 1981).

[Ka] N. J. Kalton, 'Schauder decompositions of locally convex spaces', Math. Proc. Cambridge Philos. Soc. 68 (1970), 377-392.

[Ke] J. L. Kelley, General topology (Springer, Berlin, 1975).

[Kö] G. Köthe, Topological vector spaces I, II (Springer, Berlin, 1969, 1979).

[Me] G. Meyers, 'On Toeplitz sections in F K-spaces', Studia Math. 51 (1974), 23-33.

[Mi] M. A. Miñarro, Descomposiciones de espacios de Fréchet. Aplicación al producto tensorial proyectivo (Ph.D. Thesis, Universidad de Sevilla, Seville, 1991), (in Spanish).

[PSV1] P. J. Paúl, C. Sáez and J. M. Virués, 'Completeness of spaces with Toeplitz decompositions', Rev. Real Acad. Cienc. Exact. Fís. Natur. Madrid 92 (1998), 27-33.

[PSV2] _ - 'Barrelledness of spaces with Toeplitz decompositions', preprint, 1998. 
[PeB] P. Pérez Carreras and J. Bonet, Barrelled locally convex spaces, Mathematics Studies, vol. 131 (North-Holland, Amsterdam, 1987).

[Pr] A. Peris, 'Quasinormable spaces and the problem of topologies of Grothendieck', Ann. Acad. Sci. Fenn. Ser. A I Math. 19 (1994), 167-203.

[Ru] W. H. Ruckle, Sequence spaces, Research Notes in Math., vol. 49 (Pitman, London, 1981).

[Sil] I. Singer, 'Basic sequences and reflexivity of Banach spaces', Studia Math. 21 (1961/1962), 351-369.

[Si2] _ Bases in Banach spaces I, II (Springer, Berlin, 1970, 1981).

[T] J. Taskinen, 'The projective tensor products of Fréchet-Montel spaces', Studia Math. 91 (1988), 17-30.

[V] M. Valdivia, Topics in locally convex spaces, Math. Studies, vol. 67 (North-Holland, Amsterdam, 1982).

[We] J. H. Webb, 'Schauder basis and decompositions in locally convex spaces', Math. Proc. Cambridge Philos. Soc. 76 (1974), 145-152.

[Wi1] A. Wilansky, Modern methods in topological vector spaces (McGraw-Hill, New York, 1978).

[Wi2] - Summability through functional analysis, Math. Studies, vol. 85 (North-Holland, Amsterdam, 1984).

[Ze] K. Zeller, 'Approximation in Wirkfeldern von Summierungsverfahren', Arch. Math. (Basel) 4 (1953), 425-431.

Escuela Superior de Ingenieros

Camino de los Descubrimientos s/n

41092-Sevilla

Spain

e-mail: piti@cica.es, virues@matinc.us.es 